\title{
Serum Alanine Aminotransferase As An Early Marker of Outcomes in Patients Receiving Immuno- Oncology Drugs
}

Takeshi Azuma ( $\nabla$ tazuma-tky@umin.ac.jp )

Tokyo Metropolitan Tama Medical Center

Takumi Takeuchi

Kanto Rosai Hospital

Yukihide Matayoshi

Tomishiro Central Hospital

Shin Namiki

Tokyo Metropolitan Tama Medical Center

Tetsuya Obara

Tokyo Metropolitan Tama Medical Center

Kazuhiro Imamura

Tokyo Metropolitan Tama Medical Center

Mikio Takamori

Tokyo Metropolitan Tama Medical Center

\section{Research Article}

Keywords: Immunotherapy, Immuno-onlogy drug, Nivolumab, Pembrolizumab, Iplimumab, Advanced cancer, Response predictive factor

Posted Date: February 2nd, 2021

DOI: https://doi.org/10.21203/rs.3.rs-155568/v1

License: (a) (1) This work is licensed under a Creative Commons Attribution 4.0 International License. Read Full License 


\section{Abstract}

Immune-oncology (IO) drug therapy is effective against various types of cancer. Although several, potential, clinical, predictive markers have been identified, none so far have proven reliable. Herein we evaluated changes in serum alanine aminotransferase (ALT), which is upregulated by the accumulation of activated CD8 $+T$ cells in the liver, as a potentially reliable predictive marker. We retrospectively analyzed 265 patients with advanced malignancies at three institutions between 2016 and 2019. The patients received IO drug therapy. We defined the ALT ratio (ALR) as the serum ALT value at baseline / the highest serum ALT during IO drug therapy, then determined whether the ALR correlated with the objective response rate or progression-free survival. The median follow-up was 3.1 months. We observed objective responses in 65 patients. The ALR ranged from 0.19 to 32.2 (median 1.5), and a significant ALR increase was observed in responders $(p<0.001)$. In receiver operating characteristic analysis, ALR $=1.55$ had the highest sensitivity and specificity. The patients with ALR $<1.55$ had a significantly poorer PFS than those with $A L R \geq 1.55$. A high ALR was associated with a tumor response and good PFS in patients with advanced malignancies. The ALR is a reliable predictive marker based on activated cytotoxic $T$ lymphocyte dynamics.

\section{Introduction}

Programmed death-1 (PD-1) is inducibly expressed by activated cytotoxic T lymphocytes (CTLs). PD-1 interacts with its ligand, $\mathrm{B} 7-\mathrm{H} 1$, to deliver an inhibitory signal to $\mathrm{CTLs} \mathrm{s}^{1,2}$. Cytotoxic $\mathrm{T}$ lymphocyteassociated antigen 4 (CTLA-4) is also inducibly expressed on CTLs and interacts with its ligand, CD80 or CD86, to deliver an inhibitory signal to CTLs ${ }^{3,4}$. Both molecules inhibit antitumor immunity. Anti-PD-1 and anti-CTLA-4 antibody block the inhibitory signal, thereby activating the CTLs against the cancer ${ }^{5-8}$. Recently, anti-PD-1 and anti-CTLA-4 antibodies were approved for use against several cancers ${ }^{9,10}$. These antibodies are comprehensively referred to as immune-oncology (IO) drugs. Nivolumab was effective against non-small-cell lung cancer (NSCLC) ${ }^{11,12}$, melanoma ${ }^{13}$, renal cell carcinoma (RCC) ${ }^{14}$, urothelial carcinoma (UC) ${ }^{15,16}$, squamous cell carcinoma of the head and neck (SCCHN) ${ }^{17}$, gastric cancer (GC) ${ }^{18}$, malignant mesothelioma (MM) ${ }^{19}$, Hodgkin's lymphoma ${ }^{20}$, and hepatocellular carcinoma ${ }^{21}$.

Pembrolizumab, another anti-PD-1 antibody, was also effective against NSCLC ${ }^{22}$, melanoma ${ }^{23}$, RCC $^{24}$, SCCHN ${ }^{25}, \mathrm{UC}^{26}$, and Merkel cell carcinoma ${ }^{27}$. Iplimumab was effective against melanoma ${ }^{10}$ and RCC 28.

Despite their efficacy, IO drugs benefit only a small number of patients. Therefore, reliable predictive markers allowing the identification of responders and non-responders is crucial to choosing the optimal treatment. Several studies have discussed predictive markers, such as PD-L1 expression ${ }^{29}$, overall mutational burden ${ }^{30}$, leukocyte count ${ }^{31-33}$, lactate dehydrogenase ${ }^{33,34}$, C-reactive protein ${ }^{35}$ and adverse events ${ }^{36-38}$, but as yet, no reliable predictive marker has been found. 
CTLs, which are activated by 10 drugs, accumulate in the liver and undergo apoptosis ${ }^{39,40}$. Dong et al. showed a greater accumulation of CTLs in the liver of B7-H1- deficient mice than in wild-type mice ${ }^{41}$. In the present study, we evaluated changes in the serum alanine aminotransferase (ALT) level, which is a marker of liver injury, as a mechanism-based predictive marker for 10 drug therapy.

\section{Results}

\section{Patient characteristics}

In total, 265 patients ( 134 with NSCLC (92 with adenocarcinoma and 42 with SCC); 38 with GC; 52 with RCC; 35 with UC; three with MM; and three with an unknown primary cancer) were enrolled (Table 1). The median follow-up period was 3.1 months (0.2-46.3). The average age was 69.3 years (42-88), and the male: female ratio was $193: 72$. We observed objective responses in 65 patients (44 with NSCLC (26 with adenocarcinoma and 18 with SCC); four with GC, ten with RCC; five with UC; one with MM; and one with an unknown primary cancer).

\section{ALR according to the response to immunotherapy}

The ALR ranged from 0.19 to 32.2 (median 1.5). We observed a significant increase in the ALR among responders ( $p<0.001$, Figure 1$)$. The ALR was able to predict the tumor response, as determined by ROC curve sensitivity (0.85) and specificity (0.70) (Figure 2). We observed an increase in the ALR $(\geq 1.55)$ in 54 of 65 responders ( 42 of 55 patients with a PR and 10 of 10 patients with a CR) and 67 of 200 nonresponders (27 of 55 patients with SD and 40 of 145 patients with $P D$ ) (odds ratio:9.7, $p<0.0001$, Figure 3). In 67 non-responders with an elevated ALR, 12 experienced exacerbation or liver metastasis, nine experienced adverse events associated with immunotherapy (liver toxicity, etc.), and ten showed a mixed response, in which some tumors shrank, but PR was not achieved or a new metastasis occurred.

\section{Timing of the serum ALT increase in responders}

We analyzed the timing of the serum ALT increase in 52 responders (Figure 4A). The serum ALT increased in $28(54 \%)$ and 42 (81\%) patients within 30 and 60 days, respectively, following the initial drug infusion. In all the responders, a serum ALT increase occurred within three months. Next, we evaluated the differences by drug. In patients receiving nivolumab, one (4\%), 11 (40\%), and 21 (75\%) of 28 patients showed a serum ALT increase within 10,30, and 60 days, respectively, following the initial drug infusion. In patients receiving pembrolizumab, eight (35\%), 17 (71\%), and $23(100 \%)$ of 23 patients showed a serum ALT increase within 10,30, and 60 days, respectively, following the initial drug infusion. Variations in the timing of the response after the serum ALT increase were also analyzed (Figure 4B). In fourteen (27\%), $37(71 \%)$, and $44(85 \%)$ of 52 patients, the response developed within 30,60 , and 90 days, respectively, following the serum ALT increase. In patients receiving nivolumab, one (4\%), 11 (40\%), and $21(75 \%)$ of 28 patients developed the response within 10,30, and 60 days, respectively, following the initial drug infusion. In patients receiving pembrolizumab, eight (35\%), $17(71 \%)$, and $23(100 \%)$ of 23 
patients developed the response within 10,30, and 60 days, respectively, following the initial drug infusion.

\section{Progression-free survival estimated using the ALR}

Among patients with NSCLC, GC, RCC, and UC, an ALR $<1.55$ was associated with significantly poorer PFS than $A L R \geq 1.55$. The median PFS among patients with NSCLC with $A L R<1.55$ and $A L R \geq 1.55$ was 2.6 and 12 months, respectively ( $p<0.001$, Figure $5 A$ ). Subgroup analysis performed for tumor histology demonstrated that elevated ALR remained a significant prognostic factor. The median PFS in patients with adenocarcinoma with an ALR<1.55 and ALR $\geq 1.55$ was 2.9 and 11.2 months, respectively $(p=0.002$, Figure 5B). The median PFS in patients with squamous cell carcinoma with an ALR $<1.55$ and ALR $\geq 1.55$ was 1.5 and 20.3 months, respectively $(p<0.001$, Figure 5 C). Elevated ALR was a significant prognostic factor in patients with gastric cancer, RCC, and UC. The median PFS in patients with gastric cancer with an $A L R<1.55$ and $A L R \geq 1.55$ was 1.8 and 2.2 months, respectively ( $p=0.015$, Figure $5 D$ ). The median PFS in patients with RCC with an ALR $<1.55$ and $A L R \geq 1.55$ was 4.1 months and not achieved, respectively $(p=0.006$, Figure 5E). The median PFS in patients with UC with an ALR<1.55 and ALR $\geq 1.55$ was 1.4 and 6.5 months, respectively $(p<0.001$, Figure 5F).

\section{Discussion}

Crispe et al. revealed that CTLs activated by an antigen were deleted in the liver, suggesting either a preferential accumulation in the liver of activated CTLs undergoing apoptosis (the graveyard hypothesis) or a trap for activated CTLs in the liver for subsequent killing (the killing field hypothesis) ${ }^{39,40}$. In B7-H1 deficient mice, activated PD-1 + CD8 T cells accumulated in the liver ${ }^{41}$. We surmised that the accumulation and deletion of activated CTLs in the liver might impose a burden on the liver, which could induce hepatopathy and elevate serum liver enzymes ${ }^{42}$. After assessing both serum aspartate aminotransferase and ALT, we found that serum ALT was a better predictive marker (data not shown).

The timing of the serum ALT increase varies for each IO drug. In most patients receiving pembrolizumab or a combination of nivolumab and ipilimumab, the timing of serum ALT elevation was one to three weeks following the initial drug infusion. However, the timing for nivolumab was different, varying from soon after the initial infusion to later in some cases. This difference may depend on the affinity of each IO drug to the PD-1 molecule. An antibody with a strong affinity might affect the immune system sooner than an antibody with a weaker affinity; pembrolizumab has stronger affinity to the immune system than nivolumab. Usually, serum ALT rises immediately prior to the finding of tumor shrinkage on a radiologic modality. These findings suggested that ALR might serve as a predictive marker of IO drug therapy.

The present study has the limitations inherent in any retrospective analysis. The patient population varied widely. The ALR was not a perfect predictive marker and presented certain some problems, such as the occurrence of false negative and false positive cases. We observed no increase in the ALR in 11 (17.0\%) responders while we found an increase in $67(33.5 \%)$ non-responders. There are a number of possible 
explanations for the false negatives. First, our timing of ALT analysis was inadequate in that we failed to perform the analysis when the ALT had reached its highest value. Second, because injury to the liver was small, we did not observe elevations in the serum ALT levels. There are two reasons for the low level of injury to the liver. First, the shrunken tumor volume was too small to induce sufficient CTL activation to cause hepatopathy; in effect, there was insufficient injury to the liver to elevate the ALT level. Another reason for the minimal injury to the liver was very good liver function.

There are also a number of possible explanations for the false positive results. In twelve patients, a new liver metastasis or the progression of an existing liver metastasis induced a serum ALT increase. In nine patients, other reasons, for example, autoimmune hepatitis, autoimmune cholangitis, septic shock, other drug-induced hepatopathy, etc., accounted for the elevated ALT. In ten patients, despite the achievement of SD or PD according to the RECIST criteria, a portion of the tumors showed shrinkage. We were able to identify other, clinical causes of elevated ALT as well; after excluding 21 cases of elevated ALT due to other causes from the cohort and adding ten cases showing some tumor shrinkage to the responder group, the odds ratio rose from 9.7 to 16.8 . In clinical practice, the ALR may serve as a very promising predictor of the response to 10 drug therapy when transitioning to the next treatment.

The PFS duration was significantly longer in the high ALR group than in the low ALR group for four types of cancer, suggesting that the ALR can help differentiate between long and short SD among nonresponders. The ALR is useful for patients receiving cancer immunotherapy because it facilitates judging whether immunotherapy should be continued or switched to an alternative treatment. Previous studies have reported several, reliable predictive markers for IO drug therapy, including PD-L1 expression and tumor mutation burden ${ }^{43}$. However, these factors have limitations due to cost-related concerns; these markers require additional analysis, the involvement of specialists or the use of expensive equipment. In contrast to those markers, some studies revealed that certain blood and clinical markers can be routinely used, such as the leukocyte count, lactate dehydrogenase, and adverse events ${ }^{43}$. ALR is also one such available blood markers. However, in this respect the ALR, based on CTL dynamics, is a superior marker of immunotherapy and has the potential to serve as a simple, reliable prognostic marker in risk stratification and provide better treatment allocation in cancer patients.

A high ALR was associated with the tumor response and PFS in patients with various types of cancer. An increase in ALT levels during 10 drug therapy is reliable because it is based on activated CTL dynamics, making the ALR an useful predictive marker. Further studies aimed at refining the use of the ALR as a predictive marker based on the mechanism of IO drugs therapy are warranted.

\section{Materials And Methods}

\section{Patients}

Between 2016 and 2019, we reviewed the medical records of 265 patients with advanced malignancies at Tokyo Metropolitan Tama Medical Center, Kanto Rosai Hospital, and Tomishiro-Chuo Hospital, who 
received single-agent nivolumab, pembrolizumab or a combination nivolumab and ipilimumab treatment. The study was approved by the ethical review board of Tama Medical Center, Kanto Rosai Hospital and Tomishiro Central Hospital as well as was conducted in accordance with the principles of the Declaration of Helsinki and Good Clinical Practice Guidelines. Comprehensive informed consent was obtained from all participants.

\section{Treatment and response assessment}

Patients received nivolumab $3 \mathrm{mg} / \mathrm{kg}$ every two weeks, pembrolizumab $200 \mathrm{mg}$ every three weeks or nivolumab $240 \mathrm{mg}$ every three weeks plus ipilimumab $3 \mathrm{mg}$ per kilogram every three weeks in four doses, followed by nivolumab $240 \mathrm{mg}$ every two weeks.

All the patients underwent a blood test before starting 10 drug therapy until the end of follow-up, which was performed at intervals of one to two weeks. We defined ALR as: the serum ALT value at baseline / the highest serum ALT during 10 drug therapy.

Computed tomography or magnetic resonance imaging was performed at baseline and repeated at eight to 12 weeks intervals. The clinical tumor response during treatment was assessed using the Response Evaluation Criteria in Solid Tumors (RECIST) 1.1 based on the patients' medical records. We defined a clinical benefit as a complete response (CR), partial response (PR) or stable disease (SD). We defined progression-free survival (PFS) as the time from immunotherapy initiation to the date of progression.

\section{Statistics}

We performed statistical analyses using the JMP® software package, with $p<0.05$ indicating statistical significance. We compared continuous variables using the two-tailed unpaired Student's t-test. To predict the response to, and prognosis of, IO drug therapy, we determined the optimal cutoff value of the ALR based on the receiver operating characteristic (ROC) curve and the area under the ROC curve (AUC). We constructed the distribution of the recurrence-free survival (RFS) rate using the Kaplan-Meier method.

\section{Declarations}

\section{Author Contributions}

TA designed the present study and critically revised the manuscript. TA, TT, YM, SN, TO, K.I. and M.T. performed data collection. TA analyzed the data. TA wrote the manuscript. TA, TT, YM, SN, TO, K.I. and M.T. revised the manuscript. All the authors read and approved the final manuscript.

\section{Conflicts of Interest}

All the authors state that they have no conflicts of interest.

\section{References}


1. Dong, H., Zhu, G., Tamada, K. \& Chen, L. B7-H1, a third member of the B7 family, co-stimulates T-cell proliferation and interleukin-10 secretion. Nat Med. 5, 1365-1369 https://doi.org/10.1038/70932 (1999).

2. Ishida, Y., Agata, Y., Shibahara, K. \& Honjo, T. Induced expression of PD-1, a novel member of the immunoglobulin gene superfamily, upon programmed cell death. EMBO J.11, 3887-3895 (1992).

3. Salomon, B. \& Bluestone, J. A. Complexities of CD28/B7: CTLA-4 costimulatory pathways in autoimmunity and transplantation. Annu Rev Immunol. 19, 225-252 https://doi.org/10.1146/annurev.immunol.19.1.225 (2001).

4. Melero, I., Hervas-Stubbs, S., Glennie, M., Pardoll, D. M. \& Chen, L. Immunostimulatory monoclonal antibodies for cancer therapy. Nat Rev Cancer. 7, 95-106 https://doi.org/10.1038/nrc2051 (2007).

5. Hirano, F. et al. Blockade of B7-H1 and PD-1 by monoclonal antibodies potentiates cancer therapeutic immunity. Cancer Res. 65, 1089-1096 (2005).

6. Azuma, T. et al. B7-H1 is a ubiquitous antiapoptotic receptor on cancer cells. Blood. 111, 3635-3643 https://doi.org/10.1182/blood-2007-11-123141 (2008).

7. Chen, L. et al. B7-H1 maintains the polyclonal T cell response by protecting dendritic cells from cytotoxic T lymphocyte destruction. Proc Natl Acad Sci U S A. 115, 3126-3131 https://doi.org/10.1073/pnas.1722043115 (2018).

8. O'Day, S. J., Hamid, O. \& Urba, W. J. Targeting cytotoxic T-lymphocyte antigen-4 (CTLA-4): a novel strategy for the treatment of melanoma and other malignancies. Cancer. 110, 2614-2627 https://doi.org/10.1002/cncr.23086 (2007).

9. Topalian, S. L. et al. Safety, activity, and immune correlates of anti-PD-1 antibody in cancer. NEngl J Med. 366, 2443-2454 https://doi.org/10.1056/NEJMoa1200690 (2012).

10. Hodi, F. S. et al. Improved survival with ipilimumab in patients with metastatic melanoma. $N$ Engl J Med. 363, 711-723 https://doi.org/10.1056/NEJMoa1003466 (2010).

11. Borghaei, H. et al. Nivolumab versus Docetaxel in Advanced Nonsquamous Non-Small-Cell Lung Cancer. N Engl J Med. 373, 1627-1639 https://doi.org/10.1056/NEJMoa1507643 (2015).

12. Brahmer, J. et al. Nivolumab versus Docetaxel in Advanced Squamous-Cell Non-Small-Cell Lung Cancer. N Engl J Med. 373, 123-135 https://doi.org/10.1056/NEJMoa1504627 (2015).

13. Weber, J. S. et al. Nivolumab versus chemotherapy in patients with advanced melanoma who progressed after anti-CTLA-4 treatment (CheckMate 037): a randomised, controlled, open-label, phase 3 trial. Lancet Oncol. 16, 375-384 https://doi.org/10.1016/S1470-2045(15)70076-8 (2015).

14. Motzer, R. J. et al. Nivolumab versus Everolimus in Advanced Renal-Cell Carcinoma. N Engl J Med. 373, 1803-1813 https://doi.org/10.1056/NEJMoa1510665 (2015).

15. Sharma, P. et al. Nivolumab monotherapy in recurrent metastatic urothelial carcinoma (CheckMate 032): a multicentre, open-label, two-stage, multi-arm, phase 1/2 trial. Lancet Oncol. 17, 1590-1598 (2016). 
16. Sharma, P. et al. Nivolumab in metastatic urothelial carcinoma after platinum therapy (CheckMate 275): a multicentre, single-arm, phase 2 trial. Lancet Oncol. 18, 312-322 https://doi.org/10.1016/S1470-2045(17)30065-7 (2017).

17. Ferris, R. L. et al. Nivolumab for Recurrent Squamous-Cell Carcinoma of the Head and Neck. N Engl J Med. 375, 1856-1867 https://doi.org/10.1056/NEJMoa1602252 (2016).

18. Kang, Y. K. et al. Nivolumab in patients with advanced gastric or gastro-oesophageal junction cancer refractory to, or intolerant of, at least two previous chemotherapy regimens (ONO-4538-12, ATTRACTION-2): a randomised, double-blind, placebo-controlled, phase 3 trial. Lancet. 390, 2461$2471 \mathrm{https} / / /$ doi.org/10.1016/S0140-6736(17)31827-5 (2017).

19. Scherpereel, A. et al. Nivolumab or nivolumab plus ipilimumab in patients with relapsed malignant pleural mesothelioma (IFCT-1501 MAPS2): a multicentre, open-label, randomised, non-comparative, phase 2 trial. Lancet Oncol. 20, 239-253 https://doi.org/10.1016/S1470-2045(18)30765-4 (2019).

20. Ansell, S. M. et al. PD-1 blockade with nivolumab in relapsed or refractory Hodgkin's lymphoma. $N$ Engl J Med. 372, 311-319 https://doi.org/10.1056/NEJMoa1411087 (2015).

21. El-Khoueiry, A. B. et al. Nivolumab in patients with advanced hepatocellular carcinoma (CheckMate 040): an open-label, non-comparative, phase $1 / 2$ dose escalation and expansion trial. Lancet. 389, 2492-2502 https://doi.org/10.1016/S0140-6736(17)31046-2 (2017).

22. Reck, M. et al. Pembrolizumab versus Chemotherapy for PD-L1-Positive Non-Small-Cell Lung Cancer. N Engl J Med. 375, 1823-1833 https://doi.org/10.1056/NEJMoa1606774 (2016).

23. Robert, C. et al. Pembrolizumab versus Ipilimumab in Advanced Melanoma. N Engl J Med. 372, 2521-2532 https://doi.org/10.1056/NEJMoa1503093 (2015).

24. Rini, B. I. et al. Pembrolizumab plus Axitinib versus Sunitinib for Advanced Renal-Cell Carcinoma. $N$ Engl J Med. 380, 1116-1127 https://doi.org/10.1056/NEJMoa1816714 (2019).

25. Bauml, J. et al. Pembrolizumab for Platinum- and Cetuximab-Refractory Head and Neck Cancer: Results From a Single-Arm, Phase II Study. J Clin Oncol. 35, 1542-1549 https://doi.org/10.1200/JC0.2016.70.1524 (2017).

26. Bellmunt, J. et al. Pembrolizumab as Second-Line Therapy for Advanced Urothelial Carcinoma. $N$ Engl J Med. 376, 1015-1026 https://doi.org/10.1056/NEJMoa1613683 (2017).

27. Nghiem, P. T. et al. PD-1 Blockade with Pembrolizumab in Advanced Merkel-Cell Carcinoma. N Eng/ J Med. 374, 2542-2552 https://doi.org/10.1056/NEJMoa1603702 (2016).

28. Motzer, R. J. et al. Nivolumab plus Ipilimumab versus Sunitinib in Advanced Renal-Cell Carcinoma. N Engl J Med. 378, 1277-1290 https://doi.org/10.1056/NEJMoa1712126 (2018).

29. Davis, A. A. \& Patel, V. G. The role of PD-L1 expression as a predictive biomarker: an analysis of all US Food and Drug Administration (FDA) approvals of immune checkpoint inhibitors. $J$ Immunother Cancer. 7, 278 https://doi.org/10.1186/s40425-019-0768-9 (2019).

30. Le, D. T. et al. PD-1 Blockade in Tumors with Mismatch-Repair Deficiency. N Engl J Med. 372, 25092520 https://doi.org/10.1056/NEJMoa1500596 (2015). 
31. Gebhardt, C. et al. Myeloid Cells and Related Chronic Inflammatory Factors as Novel Predictive Markers in Melanoma Treatment with Ipilimumab. Clin Cancer Res. 21, 5453-5459 https://doi.org/10.1158/1078-0432.CCR-15-0676 (2015).

32. Martens, A. et al. Baseline Peripheral Blood Biomarkers Associated with Clinical Outcome of Advanced Melanoma Patients Treated with Ipilimumab. Clin Cancer Res. 22, 2908-2918 https://doi.org/10.1158/1078-0432.CCR-15-2412 (2016).

33. Nakamura, Y. et al. Nivolumab for advanced melanoma: pretreatment prognostic factors and early outcome markers during therapy. Oncotarget. 7, 77404-77415 https://doi.org/10.18632/oncotarget.12677 (2016).

34. Diem, S. et al. Serum lactate dehydrogenase as an early marker for outcome in patients treated with anti-PD-1 therapy in metastatic melanoma. Br J Cancer. 114, 256-261 https://doi.org/10.1038/bjc.2015.467 (2016).

35. Simeone, E. et al. Immunological and biological changes during ipilimumab treatment and their potential correlation with clinical response and survival in patients with advanced melanoma. Cancer Immunol Immunother. 63, 675-683 https://doi.org/10.1007/s00262-014-1545-8 (2014).

36. Hua, C. et al. Association of Vitiligo With Tumor Response in Patients With Metastatic Melanoma Treated With Pembrolizumab. JAMA Dermatol. 152, 45-51 https://doi.org/10.1001/jamadermatol.2015.2707 (2016).

37. Rogado, J. et al. Immune-related adverse events predict the therapeutic efficacy of anti-PD-1 antibodies in cancer patients. Eur J Cancer. 109, 21-27 https://doi.org/10.1016/j.ejca.2018.10.014 (2019).

38. Weber, J. S. et al. Safety Profile of Nivolumab Monotherapy: A Pooled Analysis of Patients With Advanced Melanoma. J Clin Oncol. 35, 785-792 https://doi.org/10.1200/JC0.2015.66.1389 (2017).

39. Crispe, I. N. Hepatic T cells and liver tolerance. Nat Rev Immunol. 3, 51-62 https://doi.org/10.1038/nri981 (2003).

40. Crispe, I. N., Dao, T., Klugewitz, K., Mehal, W. Z. \& Metz, D. P. The liver as a site of T-cell apoptosis: graveyard, or killing field? Immunol Rev. 174, 47-62 https://doi.org/10.1034/j.16000528.2002.017412.x (2000).

41. Dong, H. et al. B7-H1 determines accumulation and deletion of intrahepatic CD8(+) T lymphocytes. Immunity. 20, 327-336 https://doi.org/10.1016/s1074-7613(04)00050-0 (2004).

42. Gowda, S. et al. A review on laboratory liver function tests. Pan Afr Med J. 3, 17 (2009).

43. Hopkins, A. M. et al. Predicting response and toxicity to immune checkpoint inhibitors using routinely available blood and clinical markers. Br J Cancer. 117, 913-920 https://doi.org/10.1038/bjc.2017.274 (2017).

\section{Tables}

Due to technical limitations, table 1 is only available as a download in the Supplemental Files section. 
Figures

Figure 1

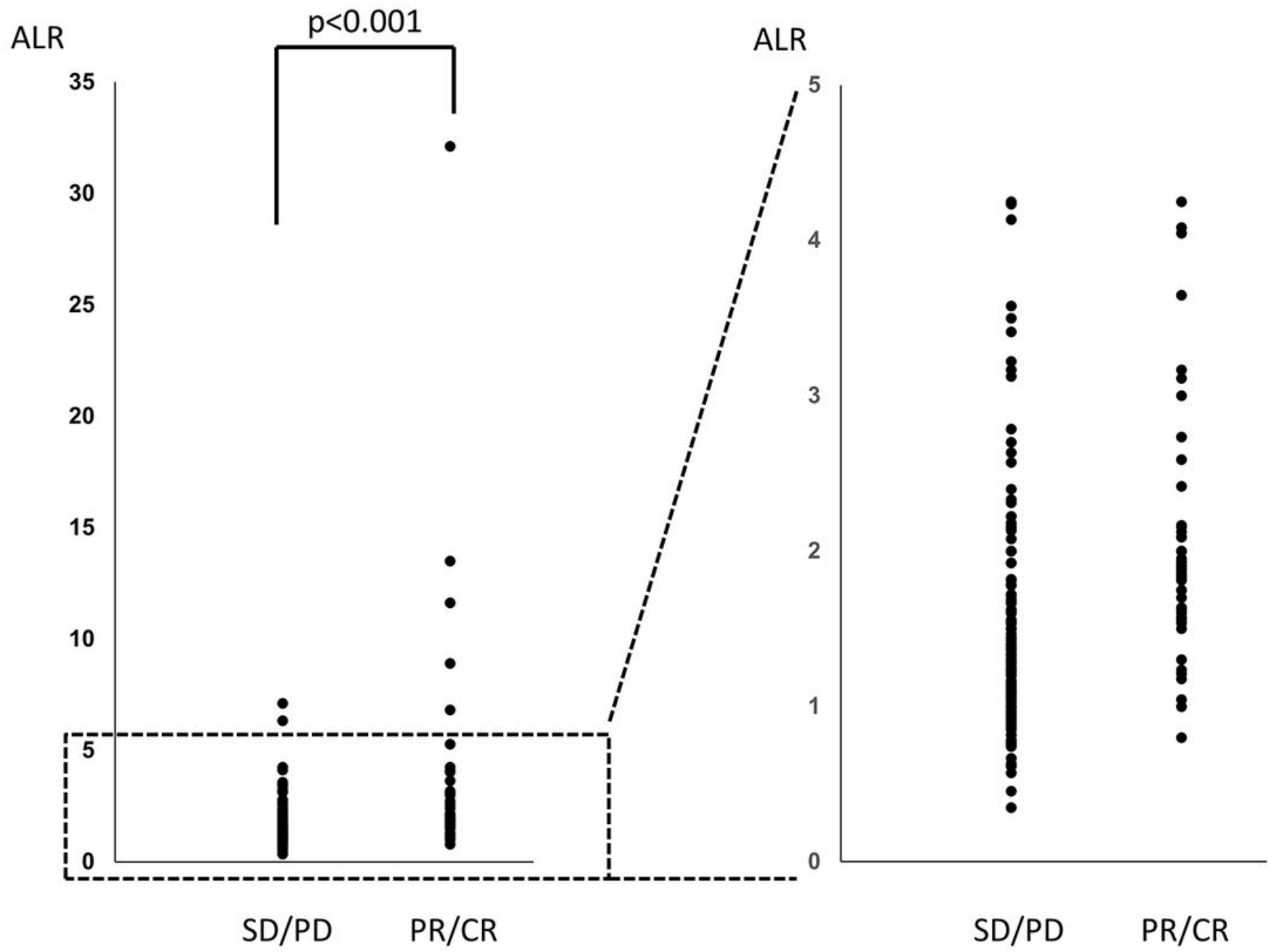

Figure 1

ALR according to the response to immunotherapy (SD/PD, stable/progressive disease; PR/CR, partial/complete response) 
Figure 2

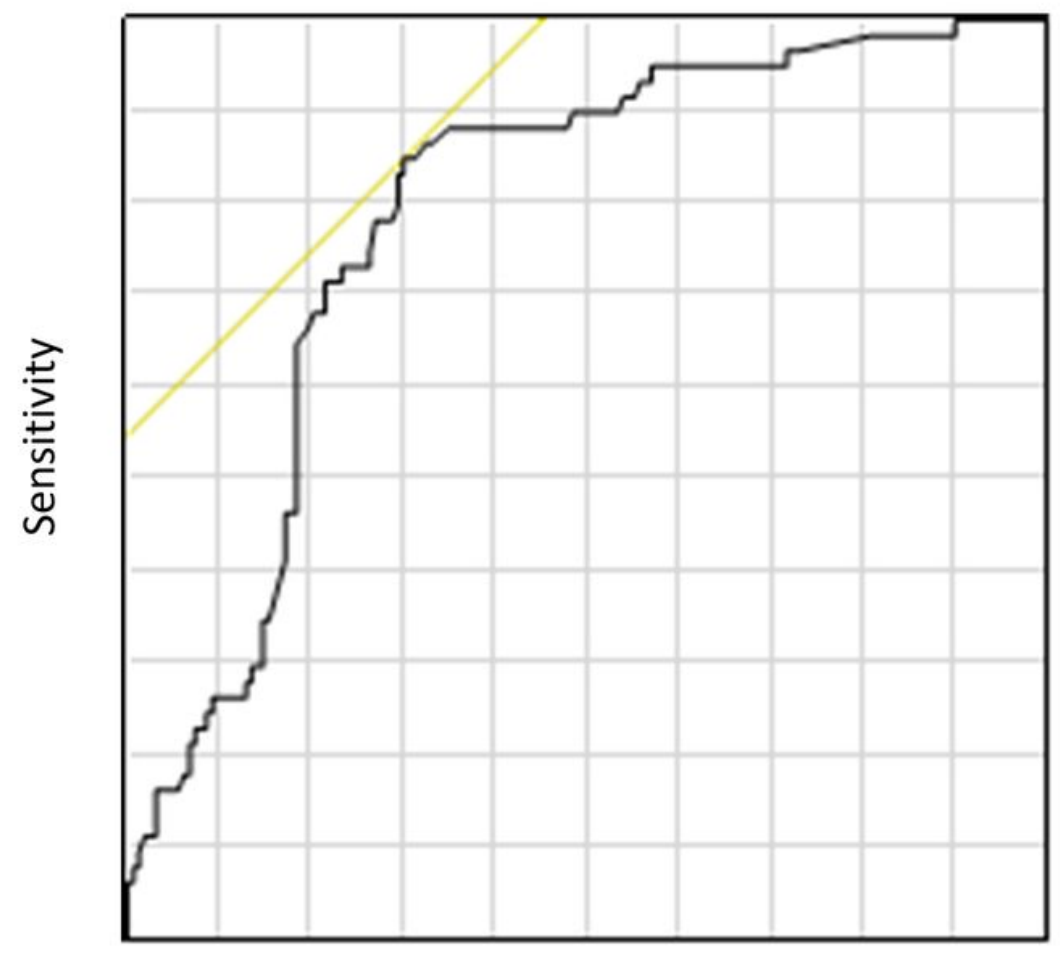

1-Specificity

\section{Figure 2}

Receiver operating curve (ROC) analysis based on the ALR for the tumor response. In this model, the sensitivity was $85.0 \%$, the specificity was $70.0 \%$, and the AUC was 0.790 . $p<0.001$. AUC, area under the curve. 
Figure 3

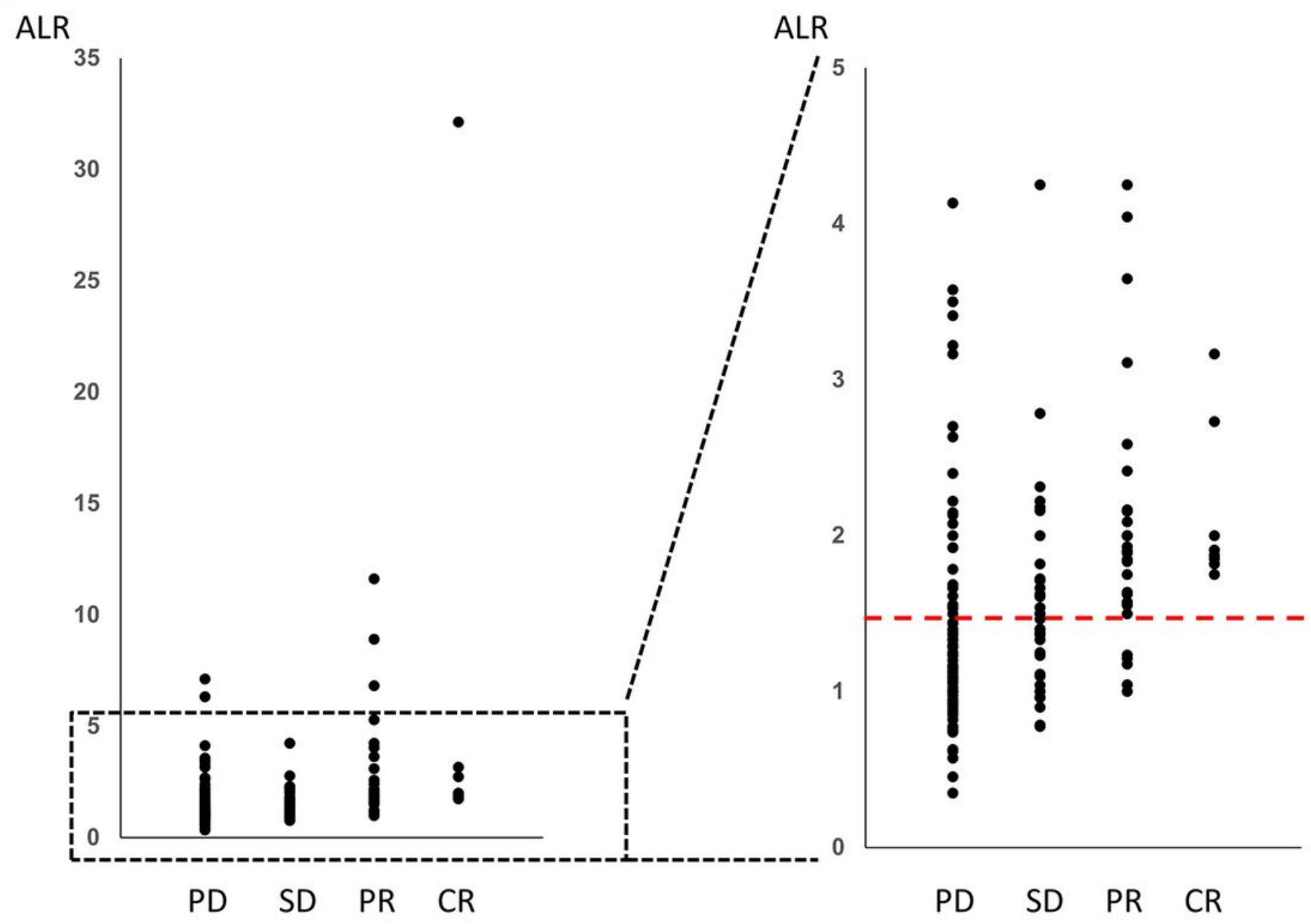

Figure 3

ALR according to the response to immunotherapy (CR, complete response; PR, partial response; SD, stable disease; PD, progressive disease) 
Figure 4A
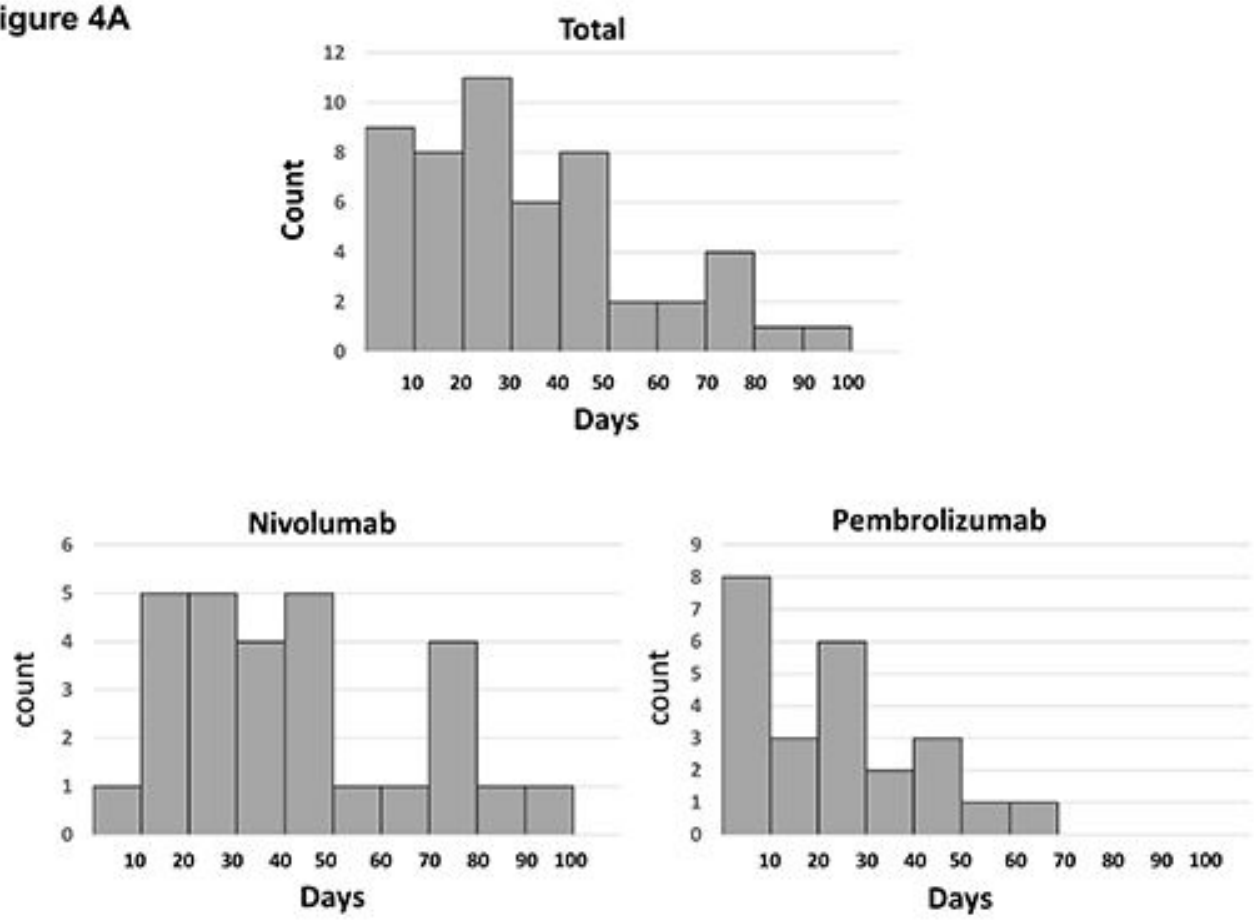

Figure 4B
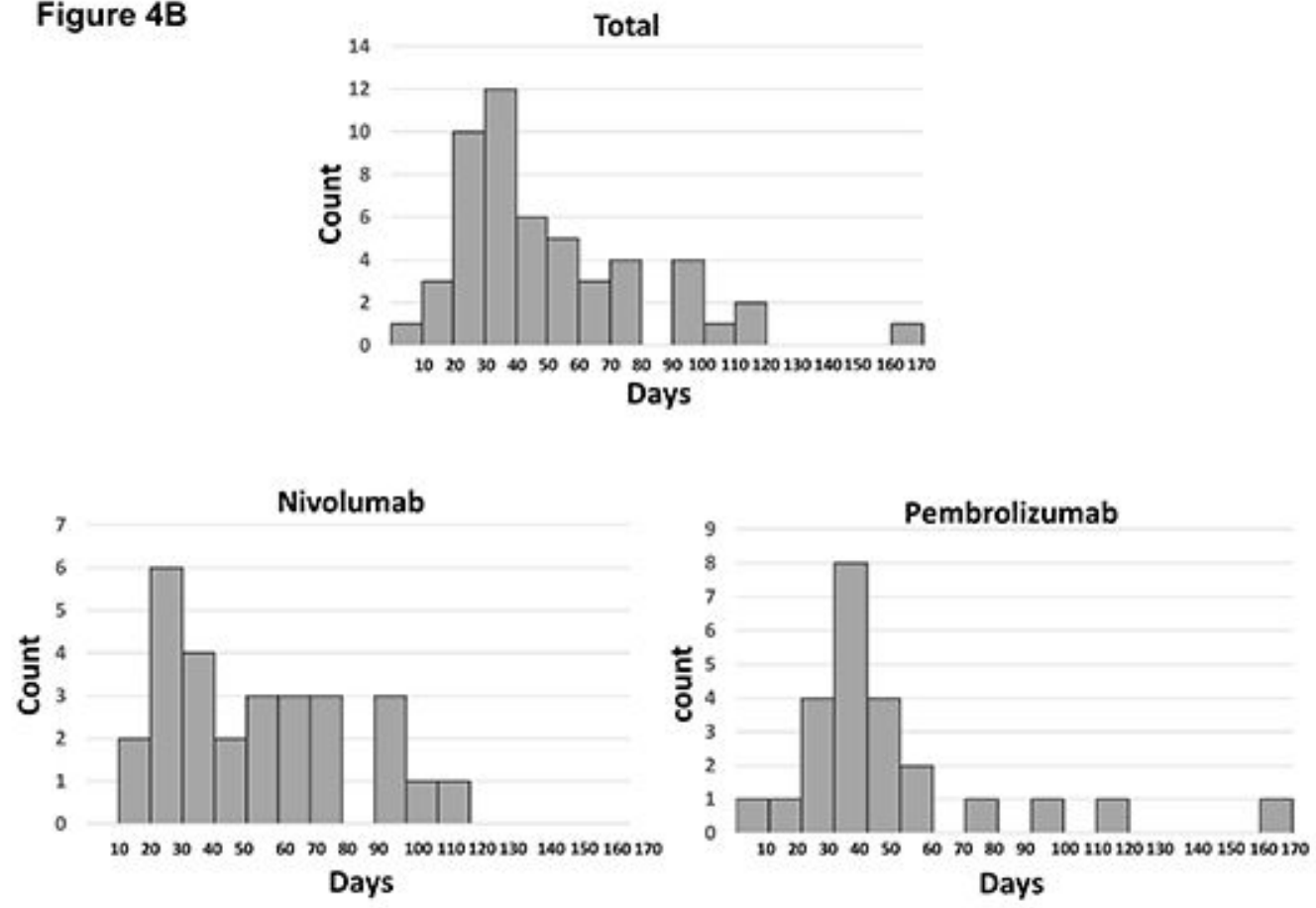

Figure 4

Timing of the serum ALT increase in responders. 

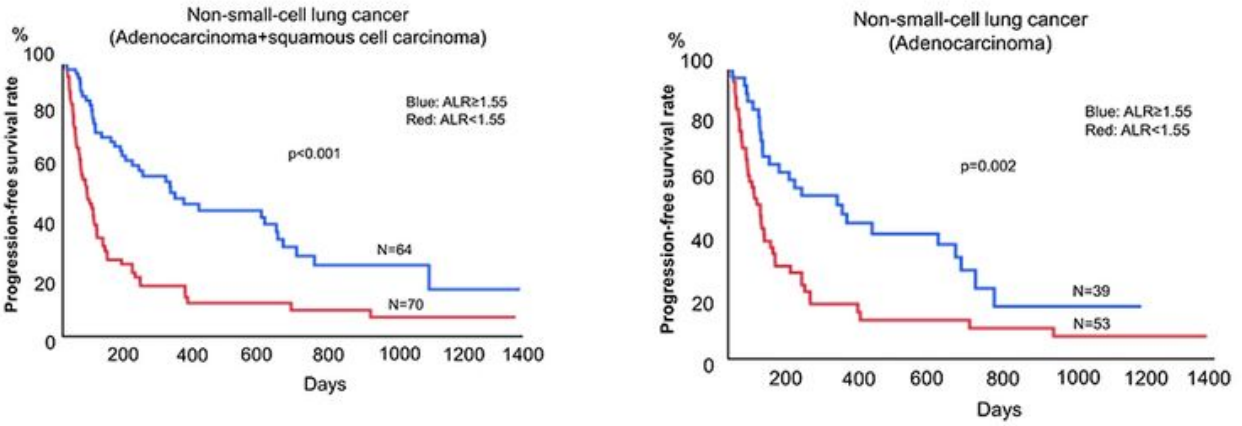

Figure $5 \mathrm{C}$

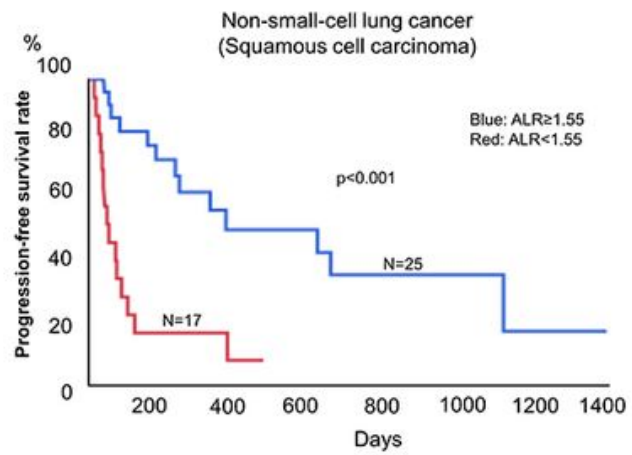

Figure 5D

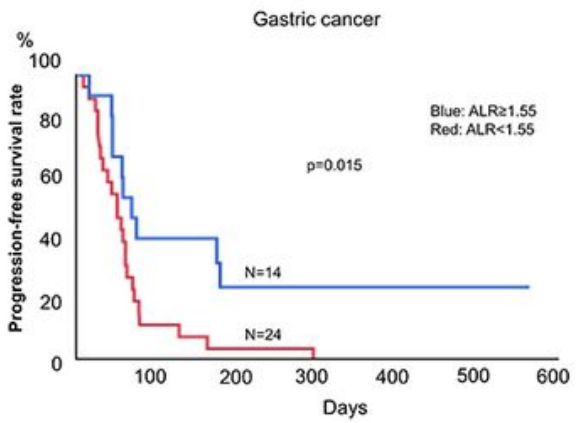

Figure 5E

Figure $5 \mathrm{~F}$
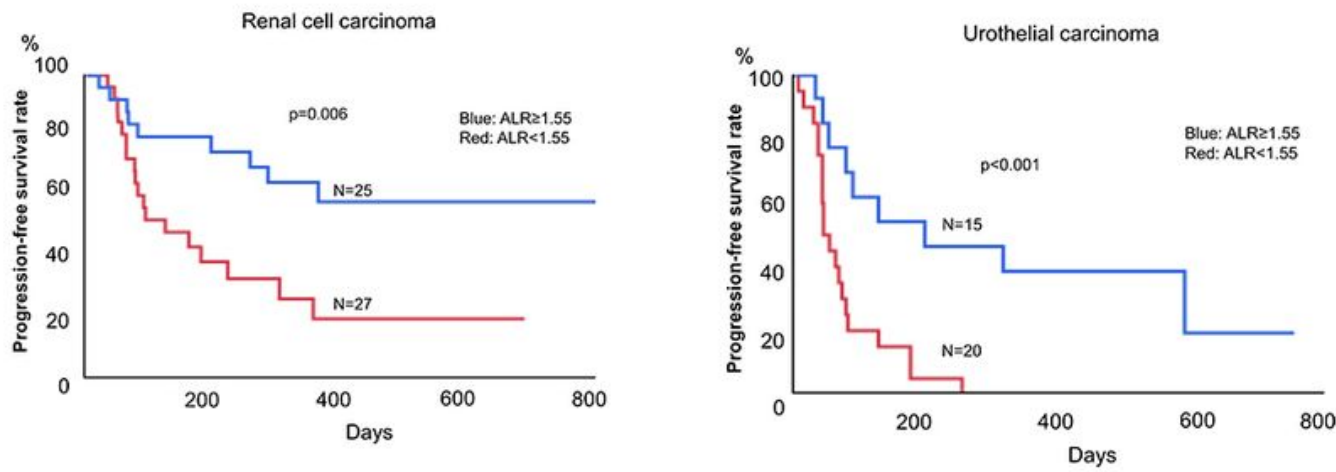

Figure 5

Progression-free survival (PFS) estimated using the ALR for non-small cell lung cancer (A), adenocarcinoma of the lung (B), squamous cell carcinoma of the lung (C), gastric cancer (D), renal cell carcinoma $(E)$ and urothelial carcinoma $(F)$. 
This is a list of supplementary files associated with this preprint. Click to download.

- Table1.jpg 